\title{
Prediction of delayed graft function after renal transplantation
}

\author{
Claudio Jeldres, MD; ${ }^{*}$ Héloïse Cardinal, MD; ${ }^{\dagger}$ Alain Duclos, MD; ${ }^{*}$ Shahrokh F. Shariat, MD; ${ }^{*}$ Nazareno Suardi, \\ MD; Umberto Capitanio, MD; ${ }^{*}$ Marie-Josèe Hébert, MD; ${ }^{\dagger}$ Pierre I. Karakiewicz, MD, FRCSC*
}

\section{Abstract}

Introduction: Delayed graft function (DGF), defined as the need for dialysis during the first week after renal transplantation, is an important adverse clinical outcome. A previous model relied on 16 variables to quantify the risk of DGF, thereby undermining its clinical usefulness. We explored the possibility of developing a simpler, equally accurate and more user-friendly paradigm for renal transplant recipients from deceased donors.

Methods: Logistic regression analyses addressed the occurrence of DGF in 532 renal transplant recipients from deceased donors. Predictors consisted of recipient age, gender, race, weight, number of HLA-A, HLA-B and HLA-DR mismatches, maximum and last titre of panel reactive antibodies, donor age and cold ischemia time. Accuracy was quantified with the area under the curve. Two hundred bootstrap resamples were used for internal validation.

Results: Delayed graft function occurred in 103 patients $(19.4 \%)$ Recipient weight $(p<0.001)$, panel of reactive antibodies $(p<0.001)$, donor age $(p<0.001)$, cold ischemia time $(p=0.005)$ and HLADR mismatches $(p=0.05)$ represented independent predictors. The multivariable nomogram relying on 6 predictors was $74.3 \%$ accurate in predicting the probability of DGF.

Conclusion: Our simple and user-friendly model requires 6 variables and is at least equally accurate (74\%) to the previous nomogram $(71 \%)$. We demonstrate that DGF can be accurately predicted in different populations with this new model.

Can Urol Assoc J 2009;3(5):377-82

\section{Résumé}

Introduction : La reprise retardée de la fonction (RRF) du greffon, définie comme le besoin de recourir à la dialyse pendant la première semaine suivant une transplantation rénale, est une issue clinique indésirable importante. Un modèle proposé antérieurement reposait sur 16 variables pour quantifier le risque de RRF, diminuant ainsi son utilité clinique. Nous avons exploré la possibilité d'élaborer un paradigme simplifié et plus convivial tout en étant tout aussi précis pour les receveurs de greffons rénaux provenant de donneurs décédés.

Méthodologie : À l'aide d'analyses de régression logistique, nous avons étudié la survenue de la RRF du greffon chez 532 receveurs de greffons rénaux provenant de donneurs décédés. Les facteurs de prédiction comprenaient l'âge, le sexe, la race et le poids du receveur et le nombre de non-concordance des phénotypes HLAA, HLA-B et HLA-DR, le titre maximal et le dernier titre d'anticorps réactifs, l'âge du donneur et la période d'ischémie froide. L'exactitude a été quantifiée par la mesure de la surface sous la courbe. Deux cents rééchantillonnages par auto-amorçage ont servi à la validation interne.

Résultats : Une reprise retardée de la fonction a été observée chez 103 patients $(19,4 \%)$. Le poids du receveur $(p<0,001)$, les anticorps réactifs $(p<0,001)$, I'âge du donneur $(p<0,001)$, la période $\mathrm{d}^{\prime}$ ischémie froide $(p=0,005)$ et la non-concordance des phénotypes HLA-DR $(p=0,05)$ constituaient des facteurs de prédiction indépendants. Le nomogramme multivarié reposant sur 6 facteurs de prédiction a permis de prédire avec une exactitude de 74,3\% la probabilité de RRF.

Conclusion : Notre modèle simple et convivial nécessite 6 variables et est au moins tout aussi exact (74\%) que le nomogramme antérieur (71\%). La RRF peut être prévue avec exactitude dans différentes populations à l'aide ce nouveau modèle, tel que nous en faisons la démonstration.

\section{Introduction}

Renal transplantation (RT) represents the optimal treatment option for most patients with end-stage renal disease. ${ }^{1}$ Due to the limited availability of allograft, only a fraction of eligible recipients are offered $\mathrm{RT}^{2}$ The paucity of available allograft emphasizes the need for optimizing RT outcomes to minimize complications and adverse outcomes that could undermine the long-term function of transplanted allograft.

There are numerous clinical and health economic consequences of renal function loss, either in the short or long term. ${ }^{3,4}$ The central importance of short-term or permanent impairment of renal function has prompted numerous analyses of predictors of acute or chronic rejection. ${ }^{5,6}$ Delayed graft function (DGF), defined as the need for dialysis within the first week of transplantation, ${ }^{7}$ occurs in up to 1 in 4 RT recipients and represents 1 of the examined adverse outcomes. ${ }^{6}$ The incidence of DGF can be reduced by a variety of interventions that modify the transplantation protocol. Recently, Irish and colleagues developed a model predicting the probability of DGF that showed $71 \%$ accuracy in external validation. ${ }^{5}$ Despite its accuracy, the model is highly complex. It requires the consideration of 11 categorical and 5 continuously coded variables for predicting DGF risk. ${ }^{5}$ The relatively large number of predictors required to quantify DGF risk limits the applicability of this nomo- 
gram in clinical practice, since one or several variables might not be available.

We decided to revisit the topic of DGF risk prediction and to test whether the concept developed by Irish and colleagues can be used in a different transplant population. We also tested whether fewer variables can accurately predict the probability of DGF in renal transplant recipients from deceased donors.

\section{Methods}

\section{Patient population}

Between 1979 and 2004, 580 patients underwent RT from deceased donors at the University of Montréal Health Centre. Donor and recipient parameters were prospectively recorded and stored in a computerized database. The database, as well as this study, was approved by the Institutional Review Board. The recorded variables included the number of HLAA, HLA-B and HLA-DR mismatches, maximum and most recent titres of panel reactive antibodies, cold ischemia time, age and gender of donor and recipient, and recipient weight and race. DGF, defined as the need for dialysis within 1 week of transplantation, represented the outcome variable. Of 580 patients, 48 were excluded due to missing maximum or last titre of reactive antibodies $(n=29)$, cold ischemia time $(n=13)$ or recipient weight $(n=6)$ information. Cold ischemia time was defined as the time between aortic cross-clamping in the donor to portal or arterial revascularization in the recipient. ${ }^{5}$

\section{Statistical methods}

Univariable and multivariable logistic regression models addressed the prevalence of DGF after RT. Predictors consisted of all recorded variables in the database. All continuously coded variables were analyzed without transformation. Subsequently, cut-off values were identified using the minimum P-value approach according to Mazumdar and Glassman. ${ }^{8}$ Categorization makes it easier for clinicians to use information about the relationship between an outcome and a predictor variable. The area under the receiver operating characteristic curve was used to test the accuracy of continuously coded and categorized predictors. In

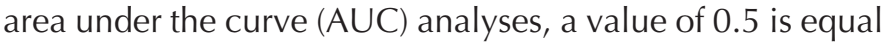
to a toss of a coin and 1.0 represents ideal predictions. Since variable categorization can inflate type I error rates, we used 200 bootstrap resamples to reduce overfit bias. ${ }^{9}$ The variable coding associated with the highest $A \cup C$ was used in the multivariable models. The full multivariable logistic regression model rested on all predictors and its 200 bootstrap-corrected AUC was quantified. Subsequently, backward variable elimination relying on Akaike's information criterion was used to identify the most parsimonious and informative combination of predictor variables. ${ }^{10,11}$ The regression-based coefficients of the predictor variables included in the most parsimonious and predictive logistic regression model were used to develop the nomogram to predict DGF after deceased donor RT. The performance characteristics of the model were graphically explored in a calibration plot. Statistical significance in this study was set at $p \leq 0.05$. All reported $p$-values were two-sided. All statistical tests were performed with S-Plus Professional (MathSoft Inc. Seattle, Washington).

\section{Results}

The descriptive statistics of the 532 assessable patients are listed in Table 1. Table 2 shows the univariable and multivariable logistic regression models that predict DGF after deceased donor RT. In univariable analyses, with the exception of the last titre of panel reactive antibodies, all continuously coded variables demonstrated higher bootstrapcorrected $A \cup C$ values after categorization. As a result, only the categorically coded formats of these variables were included in multivariable analyses (Table 2).

In univariable analyses, categorized cold ischemia time $(p=0.003)$, recipient age $(p=0.001)$, recipient weight $(p<0.001)$, HLA-DR mismatches $(p=0.01)$, maximum titre of panel reactive antibodies $(p<0.001)$ and donor age $(p<0.001)$ represented statistically significant predictors of DGF. Univariable AUC analyses revealed that donor age represented the most informative predictor of DGF (AUC 64.5\%), followed by recipient weight $(59.7 \%)$, maximal titre of panel reactive antibodies $(57.2 \%)$ and cold ischemia time (56.1\%).

In multivariable analyses, cold ischemia time $(p=0.005)$, donor age $(p<0.001)$, recipient weight $(p<0.001)$, HLADR mismatches $(p=0.05)$, maximum titre of panel reactive antibodies $(p<0.001)$ and donor age $(p<0.001)$ reached independent predictor status. The 200 bootstrap-corrected AUC of the full multivariable model was $73.1 \%$ predictive. After backward variable removal, which resulted in the final inclusion of 6 variables (cold ischemia time, recipient age, recipient weight, the number of HLA-DR mismatches, the maximal titre of panel reactive antibodies and donor age), the AUC of the most predictive and most parsimonious nomogram (Fig. 1) was $74.3 \%$ accurate.

Figure 2 shows the calibration plot of the internally validated (200 bootstraps) nomogram. The nomogram-predicted probability of DGF is shown on the x-axis and the observed rate of DGF is displayed on the $y$-axis. The bias-corrected solid line represents the nomogram performance, which approximates perfect predictions (45 degree line) with virtually no areas of under- or over-prediction. 
Table 1. Descriptive characteristics of 532 patients undergoing renal transplantation at the University of Montréal Health Centre

\begin{tabular}{|c|c|}
\hline Variables & Number (\%) \\
\hline \multicolumn{2}{|l|}{ Recipient age, yrs } \\
\hline Mean & $43(43)$ \\
\hline Range & $18-74$ \\
\hline$\leq 43$ & $272(51.1)$ \\
\hline$>43$ & 269 (48.9) \\
\hline \multicolumn{2}{|l|}{ Recipient gender } \\
\hline Male & $341(64.1)$ \\
\hline Female & $191(35.9)$ \\
\hline \multicolumn{2}{|l|}{ Recipient race } \\
\hline Caucasian & 496 (93.2) \\
\hline African-American & $14(2.6)$ \\
\hline $\begin{array}{l}\text { Other (Asian, Latin American, } \\
\text { Native American, Middle Eastern) }\end{array}$ & $22(4.2)$ \\
\hline \multicolumn{2}{|l|}{ Recipient weight, kg } \\
\hline Mean & $67.2(65.5)$ \\
\hline Range & $36-156$ \\
\hline$\leq 75$ & 393 (73.9) \\
\hline$>75$ & $139(26.1)$ \\
\hline \multicolumn{2}{|l|}{ Number of HLA-A mismatches } \\
\hline 0 & $79(14.8)$ \\
\hline 1 & $299(56.2)$ \\
\hline 2 & $154(28.9)$ \\
\hline \multicolumn{2}{|l|}{ Number of HLA-B mismatches } \\
\hline 0 & $83(15.6)$ \\
\hline 1 & $299(56.2)$ \\
\hline 2 & $150(28.2)$ \\
\hline \multicolumn{2}{|l|}{ Number of HLA-DR mismatches } \\
\hline 0 & $215(40.4)$ \\
\hline 1 & 276 (51.9) \\
\hline 2 & $41(7.7)$ \\
\hline \multicolumn{2}{|l|}{ Last titre of panel reactive antibodies, \% } \\
\hline Mean & $5.9(0)$ \\
\hline Range & $0-96$ \\
\hline \multicolumn{2}{|c|}{ Maximal titre of panel reactive antibodies, \% } \\
\hline Mean & $18.1(6)$ \\
\hline Range & $0-100$ \\
\hline$\leq 36$ & $442(83.1)$ \\
\hline $36.1-66$ & $47(8.8)$ \\
\hline$>66$ & $43(8.1)$ \\
\hline \multicolumn{2}{|l|}{ Donor age, yrs } \\
\hline Mean & $34(33)$ \\
\hline Range & $4-71$ \\
\hline \multicolumn{2}{|l|}{ Cold ischemia time, $\mathrm{h}$} \\
\hline Mean & $16.8(17)$ \\
\hline Range & $0.8-31.3$ \\
\hline$\leq 22$ & $436(82.0)$ \\
\hline $22.1-25$ & $65(12.2)$ \\
\hline$>25$ & $31(5.8)$ \\
\hline Delayed graft function, $\%$ & $103(19.4)$ \\
\hline Total & $532(100)$ \\
\hline
\end{tabular}

\section{Discussion}

Accurate identification of patients with elevated DGF risk is important, since DGF has many detrimental effects. ${ }^{6,12-14}$ Several investigators identified DGF as a predisposing factor for acute rejection and decreased graft survival. Moreover, DGF adds to the cumulative cost of RT, since it extends hospital stay, increases the number of necessary tests and obviously requires the use of dialysis facilities. ${ }^{15}$ DGF also contributes to patient anxiety. The etiology of DGF is not clear. Immunity and non-immunologic factors may play an important role. ${ }^{6,16,17}$ Their combined effects result in an increased immune response and increased organ alloreactivity.

Irish and colleagues reported a nomogram for prediction of DGF. ${ }^{5}$ The pertinence of DGF predictions was welldescribed by these authors, who identified a number of preventive measures that can reduce the probability of DGF. ${ }^{5}$ An accurate model that predicts the probability of DGF before its clinical manifestations become evident can help clinicians prevent the progression towards DGF and avoid dialysis. Several measures aimed at reducing DGF risk may be undertaken. For example, cold ischemia times might be maximally reduced and the number of HLA mismatches might be minimized. Alternatively, greater priority might be given to young recipients with low body mass indices (non-obese). Additional variables that could decrease DGF risk may be identified in institutional transplantation protocols. Several of these variables $(n=16)$ were included in the original nomogram published by Irish and colleagues. ${ }^{5}$

Identification of multiple variables that may reduce DGF risk is important from the perspective of protocol improvement since each may represent a portal for improvement of RT outcomes. However, the inclusion of 16 variables within a nomogram designed for use in busy clinical practice may not be feasible. Clinicians may find it too onerous to enter all the necessary variables and may abandon the nomogram approach. Alternatively, not all 16 predictors may be available for inclusion at all times, which may further reduce the usefulness of the nomogram. Feedback from clinicians who use nomograms ${ }^{18}$ indicates that simple nomograms are preferred over complex ones. ${ }^{19}$ Complexity refers to the number of variables and their nature. Clinicians prefer nomograms that rely on the fewest possible predictors. Moreover, clinicians prefer cut-off values to continuously coded variables to conceptualize the effect of predictor variables on the outcome of interest. ${ }^{8}$ For example, in the context of DGF, it is easier to remember that the risk is higher in recipients older than 43 years than to try conceptualizing the magnitude of the effect related to increasing age. Based on the considerations related to the complexity of the Irish nomogram, we decided to examine the possibility of developing a more user-friendly paradigm that relies on the fewest possible variables and that is coded 
Jeldres et al.

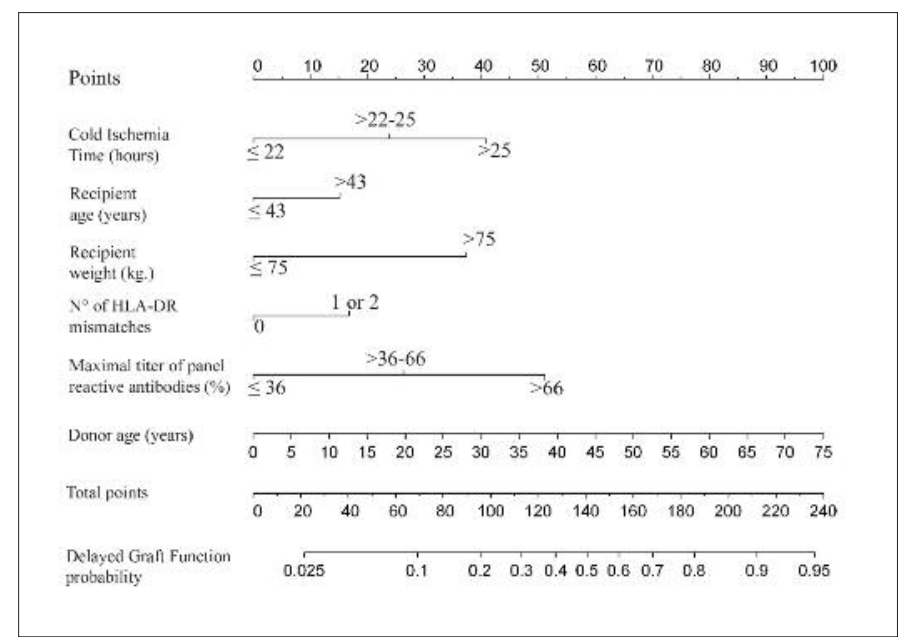

Fig. 1. Nomogram predicting the probability of delayed graft function (DGF) for patients undergoing renal transplantation from deceased donors based on cold ischemia time, recipient age and weight, number of HLA-DR mismatches, maximal titre of panel reactive antibodies and donor age. Instructions: Locate the patient's value for cold ischemia time. Draw a line straight upward to the point axis to determine how many points toward the probability of delayed graft function the patient receives for the value of cold ischemia time. Repeat the process for each additional variable. Sum the points for each of the predictors. Locate the final sum on the total point axis. Draw a line straight down to find the patient's probability of DGF.

in the most informative and intuitive fashion. Our interest was also dictated by a recent report that questioned the discriminate properties of the Irish and colleagues nomogram in a smaller-sized sample of RT recipients. ${ }^{20}$

Our analyses demonstrated that of the available predictors, 5 represented independent predictors of DGF and 1 achieved borderline predictor status. Interestingly, except for donor age, all predictors were more informative after stratification. The combination of all 6 predictors resulted in a nomogram with $74 \%$ accuracy. Since stratification of variables and the use of more informative coding schemes may inflate the rate of type I errors, ${ }^{8}$ we subjected the nomogram to an internal validation with 200 bootstrap resamples. This approach is equivalent to the application of the nomogram to 200 novel cohorts of 532 patients. Although internal validation is not synonymous with external validation, results of nomogram-derived bootstrap-corrected AUC estimates are virtually the same as externally derived ones. ${ }^{21}$ Of the 3 most frequently used internal validation methods, bootstrapping clearly emerged as superior to split-sample and crossvalidation methods. ${ }^{9}$ Indeed, Irish and colleagues demonstrated that internal validation $(71 \%)$ virtually equaled externally obtained $\mathrm{A} \cup \mathrm{C}(70 \%) .{ }^{5}$ As a result, we postulated that

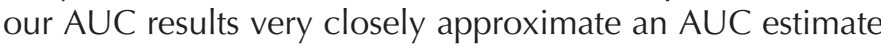
from an external validation cohort.

Interestingly, our AUC (74\%) is close to the one reported by Irish and colleagues (70\% to $71 \%) .{ }^{5}$ The similarity of accuracy estimates indicates that DGF is highly predictable across different RT populations. Moreover, despite notable

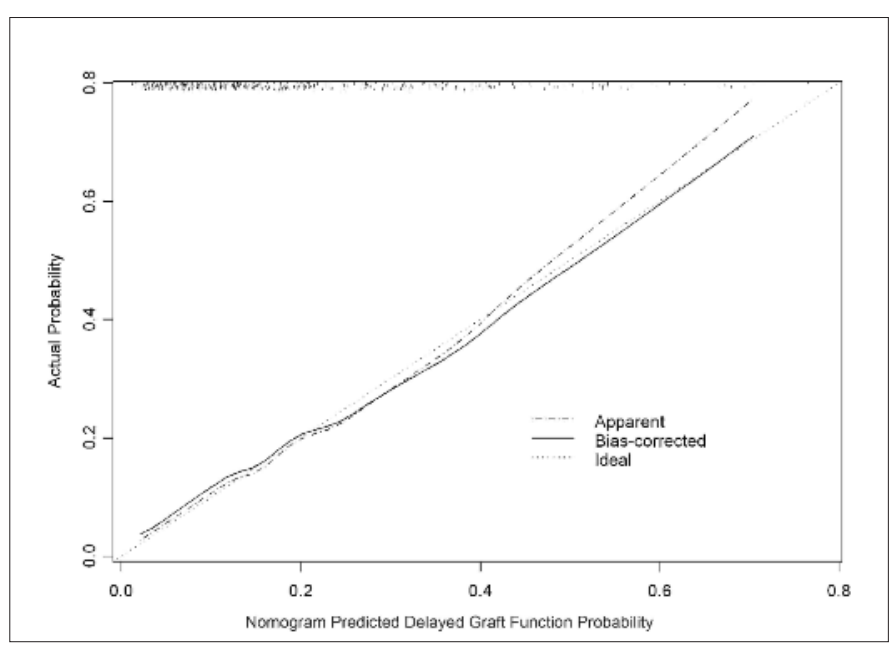

Fig. 2. Calibration plot of the newly developed nomogram. The nomogram predicting the probability of delayed graft function (DGF) is shown on the $x$-axis and the observed rate of DGF is displayed on the $y$-axis. The bias-corrected solid line represents the nomogram performance, which approximates perfect predictions ( $45^{\circ}$ line) with virtually no areas of under- or over-prediction.

population differences (e.g., different health economic contexts) the level of accuracy is also comparable. Moreover, lack of notable differences between our and the Irish and colleagues AUCs indicates that the same degree of accuracy can be expected even when the type and number of predictors differ between two study cohorts (ours: 6 predictors, vs. 16 in Irish and colleagues). Jointly, our and the Irish and colleagues' data validate the feasibility of individualized DGF predictions. As a result, the report of Grossberg and colleagues, ${ }^{20}$ which is based on a sample of 169 RT recipients, may be affected by selection biases that systematically undermine the performance of the Irish and colleagues' tool. ${ }^{5}$ Moreover, since Grossberg and colleagues do not quantify the performance of the nomogram using formal AUC methodology, it might be difficult to interpret the true merits and demerits of the Irish and colleagues' nomogram in his population. ${ }^{20}$

Our model does have limitations. First, the sample size is relatively small, especially when our data are compared to large population-based transplant registries. Unfortunately, such a registry is not yet available in Canada. Moreover, data originate from a single centre over a long period. Inclusion of additional centres and/or patients could make our findings more generalizable. Second, our dataset included some but not all predictors of DGF. For example, new immunosuppressive therapies and perioperative management strategies may also affect the current rate of DGF. Moreover, adjustment for other potential factors of DGF, such as donor morbidities, namely, kidney diseases, hypertension or diabetes, could also increase the accuracy of our nomogram and may also better represent the actual management of transplanted patients. Third, our nomogram is not perfect. Its accuracy is $74 \%$. Therefore, $26 \%$ of patients will be misclassified 


\begin{tabular}{|c|c|c|c|c|}
\hline & UVA & $\begin{array}{l}\text { UVA predictive } \\
\text { accuracy (\%) }\end{array}$ & $\begin{array}{c}\text { MVA } \\
\text { full model }\end{array}$ & $\begin{array}{l}\text { MVA } \\
\text { reduced model }\end{array}$ \\
\hline Variables & $\mathrm{RR} ; p$-value & & $\mathrm{RR} ; p$-value & \\
\hline Cold ischemia time & $-; 0.003$ & 56.1 & $-; 0.005$ & $-; 0.004$ \\
\hline $22.1-25$ vs. $\leq 22$ & $1.6 ; 0.1$ & & $2.2 ; 0.03$ & $2.1 ; 0.04$ \\
\hline$>25$ vs. $\leq 22$ & $3.5 ; 0.001$ & & $3.4 ; 0.005$ & $3.5 ; 0.004$ \\
\hline Recipient age & $-; 0.2$ & 56.2 & $-; 0.07$ & 0.06 \\
\hline$>43$ vs. $\leq 43$ & $1.7 ; 0.2$ & & $1.6 ; 0.07$ & $1.6 ; 0.06$ \\
\hline Recipient gender & $-; 0.6$ & 50.0 & $-; 0.8$ & \\
\hline Male vs. female & $0.9 ; 0.6$ & & $0.9 ; 0.8$ & \\
\hline Recipient race & $-; 0.5$ & 50.4 & $-; 0.4$ & \\
\hline Caucasian vs. African-American & $3.2 ; 0.3$ & & $3.5 ; 0.3$ & \\
\hline Others vs. African-American & $3.8 ; 0.2$ & & $5.1 ; 0.2$ & \\
\hline Recipient weight, $\mathrm{kg}$ & $-;<0.001$ & 59.7 & $-;<0.001$ & $-;<0.001$ \\
\hline$>75$ vs. $\leq 75$ & $2.5 ;<0.001$ & & $3.3 ;<0.001$ & $3.1 ;<0.001$ \\
\hline No. of HLA-A mismatches & $-; 0.4$ & 51.3 & $-; 0.6$ & \\
\hline 1 vs. 0 & $1.3 ; 0.4$ & & $1.5 ; 0.3$ & \\
\hline 2 vs. 0 & $1.6 ; 0.2$ & & $1.5 ; 0.3$ & \\
\hline No. of HLA-B mismatches & $-; 0.7$ & 50.1 & $-; 0.4$ & \\
\hline 1 vs. 0 & $0.8 ; 0.5$ & & $0.7 ; 0.2$ & \\
\hline 2 vs. 0 & $0.9 ; 0.9$ & & $0.9 ; 0.7$ & \\
\hline No. of HLA-DR mismatches & $-; 0.01$ & 56.6 & $-; 0.05$ & $-; 0.05$ \\
\hline (1 or 2 ) vs. 0 & $1.9 ; 0.01$ & & $1.7 ; 0.05$ & $1.7 ; 0.05$ \\
\hline Last titre of panel reactive antibodies, $\%$ & $1.01 ; 0.09$ & 50.1 & $0.9 ; 0.7$ & \\
\hline Maximal titre of panel reactive antibodies, \% & $-;<0.001$ & 57.2 & $-;<0.001$ & $-; 0.001$ \\
\hline $36.1-66$ vs. $\leq 36$ & $1.7 ; 0.1$ & & $2.4 ; 0.03$ & $2.2 ; 0.04$ \\
\hline$>66$ vs. $\leq 36$ & $3.6 ;<0.001$ & & $5.6 ;<0.001$ & $4.8 ;<0.001$ \\
\hline Donor age (years) & $1.03 ;<0.001$ & 64.5 & $1.04 ;<0.001$ & $1.04 ;<0.001$ \\
\hline Predictive accuracy (\%) & & & 73.1 & 74.3 \\
\hline Predictive accuracy (Mantel-Haenzel) & & & \multicolumn{2}{|c|}{$1.2 ; p<0.001$} \\
\hline Total & $532(100 \%)$ & & & \\
\hline
\end{tabular}

when our nomogram is used. Although not perfect, it predicts more accurately than the Irish and colleagues' nomogram $(70 \%$ to $71 \%) .{ }^{5}$ Moreover, nomograms have been shown to predict more accurately than clinicians. ${ }^{22}$ As a result, nomogram-derived predictions may be better than clinical intuition. Finally, it is important to emphasize that our model is only applicable to recipients from deceased donors.

\section{Conclusion}

Our simple and user-friendly model requires 6 variables and is at least equally accurate $(74 \%)$ to the previously published nomogram $(71 \%)$. This finding demonstrates that using our model can accurately predict DGF in different populations. Our nomogram with fewer variables can also readily assist clinicians in distinguishing between patients at either high or low risk of DGF after deceased donor renal transplantation.

*From the Cancer Prognostics and Health Outcome Unit, University of Montréal Health Centre, Montréal, QC; 'Department of Nephrology, University of Montréal Health Centre, Montréal, QC

Acknowledgements: Pierre I. Karakiewicz is partially supported by the University of Montréal Health Centre Urology Associates, Fonds de la Recherche en Santé du Québec, the University of Montréal Department of Surgery and the University of Montréal Health Centre (CHUM) Foundation.

This paper has been peer-reviewed. 
Jeldres et al.

Competing interests: None declared.

\section{References}

1. Greenstein S, Siegal B. Compliance and noncompliance in patients with a functioning renal transplant: a multicenter study. Transplantation 1998;66:1718-26.

2. Port FK, Merion RM, Finley MP, et al. Trends in organ donation and transplantation in the United States, 1996-2005. Am J Transplant 2007:7:1319-26.

3. Geddes CC, Woo YM, Jardine AG. The impact of delayed graft function on the long-term outcome of renal transplantation. J Nephrol 2002;15:17-21.

4. Segev DL, Gentry SE, Warren DS, et al. Kidney paired donation and optimizing the use of live donor organs. JAMA 2005;293:1883-90.

5. Irish WD, McCollum DA, Tesi RJ, et al. Nomogram for predicting the likelihood of delayed graft function in adult cadaveric renal transplant recipients. J Am Soc Nephrol 2003;14:2967-74.

6. Perico N, Cattaneo D, Sayegh MH, et al. Delayed graft function in kidney transplantation. Lancet 2004;364:1814-27.

7. Koning $\mathrm{OH}$, Ploeg RJ, van Bockel JH, et al. Risk factors for delayed graft function in cadaveric kidney transplantation: a prospective study of renal function and graft survival after preservation with University of Wisconsin solution in multi-organ donors. European Multicenter Study Group. Transplantation 1997;63:1620-8

8. Mazumdar M, Glassman JR. Categorizing a prognostic variable: review of methods, code for easy implementation and applications to decision-making about cancer treatments. Stat Med 2000;19:113-32.

9. Steyerberg EW, Harrell FE, Jr., Borsboom GJ, et al. Internal validation of predictive models: efficiency of some procedures for logistic regression analysis. J Clin Epidemiol 2001;54:774-81.

10. Atkinson AC. A note on the generalized information criterion for choice of a model. Biometrika 1980:67: 413-8.
11. Van Houwelingen IC, Le Cessie S. Predictive value of statistical models. Stat Med 1990;9:1303-25.

12. Sanfilippo F, Vaughn WK, Spees EK, et al. The detrimental effects of delayed graft function in cadaver donor renal transplantation. Transplantation 1984;38:643-8.

13. Feldman HI, Gayner R, Berlin JA, et al. Delayed function reduces renal allograft survival independent of acute rejection. Nephrol Dial Transplant 1996;11:1306-13.

14. Quiroga I, McShane P, Koo DD, et al. Major effects of delayed graft function and cold ischaemia time on renal allograft survival. Nephrol Dial Transplant 2006;21:1689-96

15. Almond PS, Troppmann C, Escobar F, et al. Economic impact of delayed graft function. Transplant Proc 1991;23:1304

16. Asher J, Wilson C, Gok M, et al. Factors predicting duration of delayed graft function in nonheart-beating donor kidney transplantation. Transplant Proc 2005;37:348-9.

17. Senel FM, Karakayali H, Moray G, et al. Delayed graft function: predictive factors and impact on outcome in living-related kidney transplantations. Ren Fail 1998;20:589-95.

18. Take the Nomogram Challenge. www.nomogram.org/full/pros_calc.php (Accessed August 31, 2009).

19. Capitanio U, Jeldres C, Shariat SF, et al. Clinicians are most familiar with nomograms and rate their clinical usefulness hghest, look-up tables are second best. Eur Urol 2008;54:958-9.

20. Grossberg JA, Reinert SE, Monaco AP, et al. Utility of a mathematical nomogram to predict delayed graft function: a single-center experience. Transplantation 2006;81:155-9.

21. Chun FK, Graefen M, Briganti A, et al. Initial Biopsy Outcome Prediction-Head-to-Head Comparison of a Logistic Regression-Based Nomogram versus Artificial Neural Network. Eur Urol 2007:51:1236-43.

22. Specht $M C$, Kattan $M W$, Gonen $M$, et al. Predicting nonsentinel node status after positive sentinel lymph biopsy for breast cancer: clinicians versus nomogram. Ann Surg Oncol 2005;12:654-9.

Correspondence: Dr. Pierre I. Karakiewicz, Associate Professor and Director, Cancer Prognostics and Health Outcome Unit, University of Montréal Health Centre (CHUM), 1058 St-Denis Street, Montréal, QC H2X 3J4; fax: 514-227-5103; pierre.karakiewicz@umontreal.ca or www.nomogram.org 